\title{
Contribution of capecitabine for therapy of patients with gastroesophageal cancer: an update of recent phase III results
}

\author{
Putao Cen \\ Eric D Tetzlaff \\ Jaffer A Ajani
}

Department of Gastrointestinal Medical Oncology in the Division of Cancer Medicine at the University of Texas MD Anderson Cancer Center, Houston, TX, USA
Correspondence: Jaffer A Ajani MD Anderson Cancer Center; Box 426, I5 I5 Holcombe Boulevard, Houston, TX 77030-4009, USA

$\mathrm{Tel}+\mathrm{I} 7137922828$

Fax + I 713745 II63

Email jajani@mdanderson.org
Background: Capecitabine, an orally administered fluoropyrimidines, is widely used in the treatment of multiple malignancies. It has been extensively evaluated in patients with gastroesophageal carcinoma. Since recent reviews have discussed phase I/II trials (Cancer 107:221-231, 2006; Drugs 67:601-610, 2007), we focus on the impact of the results of the most current phase III trials using capectiabine in the treatment of advanced gastroesophageal cancers, primarily in the first-line setting.

Methods: To find published phase III trials, Medline was searched for English-language clinical trials published from 1996 through June 2007 along with relevant abstracts presented at the American Society of Clinical Oncology, and meetings of the European Cancer Conference and European Society of Medical Oncology. Only representative trials were chosen for this manuscript.

Results: The most frequently investigated combinations are capecitabine with taxanes, platinols, and camptothecins. Recent results of a large phase III trial (REAL-2) in untreated patients with gastroesophageal cancer suggest that capecitabine is a non-inferior substitute for intravenous 5-fluorouracil. These results of REAL-2 trial are substantiated by a smaller phase III trial. Previous analysis of multiple trials had suggested that capecitabine, when combined in doses lower than $1250 \mathrm{mg} / \mathrm{m}^{2}$ twice daily, consistently resulted in lower frequency of Grade 3 or 4 toxic effects.

Conclusions: Capecitabine provides much needed convenience to patients with gastroesophageal cancer. The recent data derived from two phase III trials confirm that capecitabine is a suitable substitute for intravenous 5-fluorouracil in patients whose swallowing is not greatly affected. Capecitabine remains a subject of further investigations in this group of patients with interest.

Keywords: capecitabine, gastroesophageal cancer, oral fluoropyrimidines

\section{Introduction}

Gastroesophageal cancers represent a major health care burden throughout the world. The incidence in the USA is relatively low and has resulted in considerable lack of much needed research in this area. Adenocarcinoma involving the gastroesophageal junction and proximal stomach is on a rapid rise in the West (Phol and Welch 2005). The combined incidence of gastroesophageal cancer exceeds 1.4 million cases per year (Cancer Facts and Figures 2006) and among these gastric cancers make up the fourth most common cancer worldwide, with 934,000 new cases per year, and the second most common cause of cancer-related death, with 700,000 deaths annually (Cancer Facts and Figures 2006). 5-fluorouracil (5-FU) is one of the most commonly used drugs in patients with advanced gastroesophageal cancer. It has been used more consistently than any other class of agents. However, depending on the schedule and dose of 5-fluourouracil, patient may experience considerable toxicities. Currently, 
one stated goal for the future development of cytotoxic combinations in patients with advanced gastric cancer is to focus on therapy that is more convenient, has a favorable safety profile but without compromise of the efficacy.

Oral fluoropyrimidines have the potential to fulfill the two of the stated goals for improving the overall profile of cytotoxic combination chemotherapy. S-1, an agent not available widely, is approved for the treatment of advanced gastric cancer in Japan and Korea, and has been investigated on a limited basis in the West (Schuller et al 2000; Chollet et al 2003; Ajani et al 2005, 2006; Lenz et al 2007). Capecitabine, on the other hand, has been a subject of investigation in patients with gastroesophageal cancers for some time as reviewed recently (Ajani 2006; Dhillon and Scott 2007). Here we focus mainly on the current research involving phase III studies performed with capecitabine in combination with other agents in the treatment of patients with advanced gastroesophageal cancers.

Capecitabine is a novel, orally administered fluoropyrimidine carbamate that is readily absorbed by the gastrointestinal tract and is metabolized by the liver, where it is converted initially to $5^{\prime}$ deoxy-5-fluorocytidine (5'-DFCR) and subsequently to $5^{\prime}$ deoxy-5-fluorouridine (5'-DFUR). Designed to mimic continuous intravenous infusion of 5-fluorouracil (5-FU), oral capecitabine predominantly concentrates in tumor tissue (Roche Laboratories Inc 2005). Capecitabine is currently approved as a single agent for adjuvant treatment of Stage III colon cancer and for first-line metastatic colorectal cancer (when fluoropyrimidine therapy alone is preferred), and for metastatic breast cancer as a single agent (for patients resistant to paclitaxel and anthracyclines) and in combination with docetaxel (after failure of anthracycline-based therapy) (Hong et al 2004).

\section{Absorption and compliance}

Capecitabine is fully absorbed from the gastrointestinal tract (particularly from the small intestines) unaltered and converted to 5'-DFCR and 5'-DFUR to 5-fluorouracil (Kochi et al 2007) The absorption of oral fluoropyrimidines is unaffected by gastrectomy (Camidge et al 2005) Clearly, patients who have significant nausea and/or vomiting are suitable for capecitabine therapy. In addition, patients who have dysphagia to solids should not be recommended oral therapy, including capecitabine. Compliance to oral therapy is certainly an important issue. Compliance is highly dependent on the frequency and duration of therapy. Capecitabine is administered twice daily for 7-14 consecutive days; therefore, the compliance has not been a significant issue in these patients.

\section{Methodology}

Medline was searched for English-language clinical trials published from 1996 through June 2007 containing the terms "capecitabine" or "Xeloda" and "gastric," "stomach," "gastroesophageal" in the title or abstract. Potentially relevant abstracts presented at annual meetings or gastrointestinal symposia of the American Society of Clinical Oncology (ASCO), and from meetings of the European Cancer Conference (ECCO) and the European Society of Medical Oncology (ESMO), dating back to year 2000 were examined. Only the most recent data from reported trials were included.

The focus of this review is predominantly on efficacy in patients with advanced gastroesophageal cancer and to some extent on dose-related toxic effects. The review also focuses on mainly on combination of capecitabine with other agents and greater description of two newly reported phase III trials.

\section{Results}

Several phase II trials (Louvet et al 2002; Koizumi et al 2003; Kondo et al 2003; Sakamoto et al 2006) have evaluated the efficacy and safety of capecitabine as a single agent for treatment of gastric cancer. Capecitabine has been combined with taxanes, platinols, and camptothecins in many phase I and phase II settings, as reviewed previously (Ajani 2006; Dhillon and Scott 2007). With regards to complications related to capecitabine, it was noted from our previous review that patients with who received more than $2500 \mathrm{mg} / \mathrm{m}^{2}$ per day of capecitabine in combination with other agents, experienced a high rate of related grade 3 or 4 toxicity. Therefore, it was suggested the daily dose of capecitabine be reduced to less than $2500 \mathrm{mg} / \mathrm{m}^{2} /$ day when combined with other cytotoxic agents.

\section{Results of recent phase III studies}

Oxaliplatin when combined with 5-fluorouracil is active against gastric or gastroesophageal carcinoma (Louvet et al 2002). It has been substituted for cisplatin in a couple of recent phase III trials (Cunningham et al 2006; Kang et al 2006). In the REAL 2 trial, more than 1000 patients were randomized in a $2 \times 2$ design to epirubicin, cisplatin, and 5-fluororuacil (ECF serving as the reference regimen) or to 3 other regimens that systematically substituted oxaliplatin for cisplatin (as in epirubicin, oxaliplatin, and 5-fluorouracil [EOF] or epirubicin, oxaliplatin, and capecitabine [EOX]) or capecitabine for 5-fluorouracil (as in EOX or epirubicin, cisplatin, and capecitabine [EOX]). The primary endpoint of this trial was focused on overall survival (OS) (acceptable hazard ratio [HR] up to 1.23 ) to demonstrate the non-inferiority 
of capecitabine when substituting it for 5-fluorouracil and the non-inferiority when substituting oxaliplatin for cisplatin. The secondary endpoints were to compare all 4 regimens, review safety, and TTP. This trial has mature results. The median follow-up time is more than 17 months and more than 850 events have already occurred. Overall, the 60-day all cause mortality was in the range of $8 \%$ for all 4 regimens. Similarly, the mortality from 30 days from last chemotherapy administration was similar. One-year survival was also very similar and so was the median survival per protocol. The HR was 0.86 for the primary comparison between capecitabine and 5-fluorouracil and the HR was 0.92 for the primary comparison between oxaliplatin and cisplatin. Non-inferiority was maintained for capecitabine $(\mathrm{HR}=0.89)$ and oxaliplatin $(\mathrm{HR}=0.95)$ after multivariable analysis was performed with performance status, extent of disease, and age included in the model. In addition, there was more hand-and-foot syndrome in the capecitabine arms and there was more neuropathy in the oxaliplatin arms. Finally, all 4 regimens were generally well tolerated with, at the most, a $10 \%$ rate of complicated neutropenia. In addition, the baseline QoL was similar at baseline for all 4 arms. After starting protocol chemotherapy, QoL at 12 and 24 weeks was also similar in all 4 arms.

REAL 2 is an important trial that demonstrated that capecitabine is non-inferior to 5-fluorouracil and oxaliplatin is non-inferior to cisplatin. Capecitabine had a different toxicity profile compared to 5-FU however it was not necessarily better tolerated than 5-FU. In addition, capecitabine is probably more convenient for patients but the QoL scores are not different between treatments arms.

Kang et al (2006) have confirmed the findings of the REAL 2 trial in a multi-national phase III trial. In this recently presented trial, 316 patients with untreated advanced gastric carcinoma received infusional 5 -fluorouracil $800 \mathrm{mg} / \mathrm{m}^{2}$ on days $1-5$ and cisplatin $80 \mathrm{mg} / \mathrm{m}^{2}$ (FP) administered every 3 weeks or capecitabine $1000 \mathrm{mg} / \mathrm{m}^{2}$ twice daily for 14 days and cisplatin $80 \mathrm{mg} / \mathrm{m}^{2}$ (XP) administered every 3 weeks. The primary endpoint was to demonstrate non-inferiority when capecitabine is substituted for 5-fluorouracil in OS with acceptable HR of $<1.4$. The trial was conducted in 46 centers and 3 countries. The median follow-up time for this trial was 22 months. The HR for the primary endpoint was 0.81 (the median survival for $\mathrm{XP}=10.5$ months and that for $\mathrm{FP}=9.3 ; \mathrm{p}=0.27$ ). The toxicity profile was similar for both regimens. Summary of two recent phase III trials using capecitabine is provided in Table 1.

There was no QoL assessment in the Kang et al trial. In addition, this trial used lower doses of cisplatin and 5-FU in the reference arm as compared to other recent phase III trials with FP (Dank et al 2005; Van Cutsem et al 2006). A more standard dosing regimen of cisplatin and 5-FU may have influenced the finals results. In conclusion, this trial shows that capecitabine is non-inferior to 5-fluorouracil.

\section{Conclusions and future directions}

Capecitabine is an important agent in the treatment of advanced gastroesophageal cancer. It is one of the most investigated oral fluoropyrimidines. The results of the most recent phase III trials demonstrate that capecitabine is noninferior to intravenously administered 5-fluororuacil. The toxicity of capecitabine (particularly hand-foot syndrome and diarrhea) is dose dependent. Capecitabine is currently being studied in combination with intravenous cytotoxic and biologic agents, with radiation therapy and in the adjuvant setting. Further studies of capecitabine in combination with oral cytotoxic and oral biologic agents are warranted.

\section{Acknowledgement}

This project was supported in part by grants from the Park, Cantu, Smith, Ziffer, and Dallas families and by the Rivercreek Foundation.

Table I Summary of randomized phase III trials investigating capecitabine in advanced gastroesophageal cancer

\begin{tabular}{lllll}
\hline & Treatment & $\begin{array}{l}\text { Response rate } \\
\text { (overall) }\end{array}$ & $\begin{array}{l}\text { Median overall } \\
\text { survival (months) }\end{array}$ & $\begin{array}{l}\text { Hazard ratio } \\
\mathbf{( 9 5 \% ~ C l )}\end{array}$ \\
\hline Cunningham et al 2006 & ECF & $41 \%$ & 9.9 & 1 \\
& EOF & $42 \%$ & 9.3 & $0.95(0.79-1.15)$ \\
& ECX & $46 \%$ & 9.9 & $0.92(0.76-1.11)$ \\
Kang et al 2006 & EOX & $48 \%$ & 11.2 & $0.80(0.65-0.97)^{*}$ \\
& FC & $29 \%$ & 9.3 & 1 \\
& XC & $41 \% * *$ & 10.5 & $0.81(0.63-1.04)$ \\
\hline
\end{tabular}

$*_{\mathrm{p}}$ value $=0.025$ compared to ECF.

** $p$ value $=0.03$.

Abbreviations: C, cisplatin; $\mathrm{Cl}$, confidence interval; E, epirubicin; F, 5-fluorouracil; X, capecitabine. 


\section{References}

Ajani J. 2006. Review of capecitabine as oral treatment of gastric, gastroesophageal, and esophageal cancers. Cancer, 107:221-31.

Ajani JA, Faust J, Ikeda K, et al. 2005. Phase I pharmacokinetic study of S-1 plus cisplatin in patients with advanced gastric carcinoma. J Clin Oncol, 23:6957-65.

Ajani JA, Lee FC, Singh DA, et al. 2006. Multicenter phase II trial of S-1 plus cisplatin in patients with untreated advanced gastric or gastroesophageal junction adenocarcinoma. J Clin Oncol, 24:663-7.

Camidge R, Reigner B, Cassidy J, et al. 2005. Significant effect of capecitabine on the pharmacokinetics and pharmacodynamics of warfarin in patients with cancer. J Clin Oncol, 23:4719-25.

Cancer Facts and Figures. 2006. [online]. Accessed March 28, 2007. URL: www.cancer.org

Chollet P, Schoffski P, Weigang-Kohler K, et al. 2003. Phase II trial of S-1 in chemotherapy-naive patients with gastric cancer. A trial performed by the EORTC Early Clinical Studies Group (ECSG). Eur J Cancer, 39:1264-70.

Cunningham D, Rao S, Starling N, et al. 2006. Randomized multicenter phase III study comparing capecitabine with fluorouracil and oxaliplatin with cisplatin in patients with advanced esophagogastric cancer. The REAL 2 trial. Proc Am Soc Clin Oncol, 24:18S(Abstract no. LBA4017).

Dank M, Zaluski J, Barone C, et al. 2005. Randomized phase 3 trial of irinotecan (CPT-11) 5FU/folinic acid (FA) vs CDDP 5FU in 1st-line advanced gastric cancer patients. Proc Am Soc Clin Oncol Gastrointestinal Cancers Symposium, 2005. (Abstract 61).

Dhillon S, Scott LJ. 2007. Capecitabine: in advanced gastric or gastroesophageal cancer. Drugs, 67:601-10.

Hong YS, Song SY, Lee SI, et al. 2004. A phase II trial of capecitabine in previously untreated patients with advanced and/or metastatic gastric cancer. Ann Oncol, 15:1344-7.

Kang Y, Kang WK, Shin DB, et al. 2006. Randomized phase III trial of capecitabine/cisplatin (XP) vs. continuous infusion of 5-FU/cisplatin (FP) as first-line therapy in patients with advanced gastric cancer: efficacy and safety results. Proc Am Soc Clin Oncol, 24:18S (Abstract no. LBA 4018).
Kochi M, Fujii M, Kanamori N, et al. 2007. Effect of gastrectomy on the pharmacokinetics of S-1, an oral fluoropyrimidine, in resectable gastric cancer patients. Cancer Chemother Pharmacol, 60:693-701.

Koizumi W, Saigenji K, Ujiie S, et al. 2003. A pilot phase II study of capecitabine in advanced or recurrent gastric cancer. Oncology, 64:232-6.

Kondo K, Chin K, Sakamoto J, et al. 2003. A multicenter phase II trial using 4-week cycles of capecitabine in advanced/metastatic gastric cancer (AGC). Proc Am Soc Clin Oncol, 22:321(Abstract 1289).

Lenz H-J, Lee F-C, Haller DG, et al. 2007. Extended safety and efficacy data on S-1 plus cisplatin in patients with untreated advanced gastric carcinoma in a multi-center phase II study. Cancer, 109:33-40.

Louvet C, Andre T, Tigaud J, et al. 2002. Phase II study of oxaliplatin, fluorouracil, and folinic acid in locally advanced or metastatic gastric cancer patients. J Clin Oncol, 20:4543-8.

Pohl H, Welch HG. 2005. The role of overdiagnosis and reclassification in the marked increase of esophageal adenocarcinoma incidence. $J$ Natl Cancer Inst, 97:142-6

Roche Laboratories Inc. 2005. Xeloda (capecitabine) tablets prescribing information. [online]. Accessed September 15, 2005. URL: http://www. rocheusa.com/products/xeloda/pi pdf.

Sakamoto J, Chin K, Kondo K, et al. 2006. Phase II study of a 4-week capecitabine regimen in advanced or recurrent gastric cancer. Anticancer Drugs, 17:231-6.

Schuller J, Cassidy J, Dumont E, et al. 2000. Preferential activation of capecitabine in tumor following oral administration to colorectal cancer patients. Cancer Chemother Pharmacol, 45:291-7.

Van Cutsem E., Moiseyenko VM, Tjulandin S, et al. 2006. Phase III study of docetaxel and cisplatin plus fluorouracil compared with cisplatin and fluorouracil as first-line therapy for advanced gastric cancer: a report of the V325 Study Group. J Clin Oncol, 24:4991-7. 